Publ. Mat. 63 (2019), 423-444

DOI: 10.5565 /PUBLMAT6321902

\title{
FIVE SOLVED PROBLEMS ON RADICALS OF ORE EXTENSIONS
}

\author{
Be'eri Greenfeld, Agata Smoktunowicz, and Michą \\ ZIEMBOWSKI
}

\begin{abstract}
We answer several open questions and establish new results concerning differential and skew polynomial ring extensions, with emphasis on radicals. In particular, we prove the following results.

If $R$ is prime radical and $\delta$ is a derivation of $R$, then the differential polynomial ring $R[X ; \delta]$ is locally nilpotent. This answers an open question posed in [41].

The nil radical of a differential polynomial ring $R[X ; \delta]$ takes the form $I[X ; \delta]$ for some ideal $I$ of $R$, provided that the base field is infinite. This answers an open question posed in $[\mathbf{3 0}]$ for algebras over infinite fields.

If $R$ is a graded algebra generated in degree 1 over a field of characteristic zero and $\delta$ is a grading preserving derivation on $R$, then the Jacobson radical of $R$ is $\delta$-stable. Examples are given to show the necessity of all conditions, thereby proving this result is sharp.

Skew polynomial rings with natural grading are locally nilpotent if and only if they are graded locally nilpotent.

The power series ring $R[[X ; \sigma, \delta]]$ is well-defined whenever $\delta$ is a locally nilpotent $\sigma$-derivation; this answers a conjecture from [13], and opens up the possibility of generalizing many research directions studied thus far only when further restrictions are put on $\delta$.
\end{abstract}

2010 Mathematics Subject Classification: 16N20, 16N40, 16N60, 16N80, 16S32, 16S36, 16W50.

Key words: Skew-polynomial extensions, differential polynomial rings, Jacobson radical, graded nil algebras.

\section{Introduction}

In this paper we investigate the properties of several classes of ideals in twisted ring extensions. We are particularly interested in prime, nilpotent, and locally nilpotent ideals. Recently applications of this research

The first named author was partially supported by an ISF grant \#1623/16. The second named author was supported by ERC Advanced grant Coimbra 320974. The third named author was supported by the Polish National Science Centre grant UMO2017/25/B/ST1/00384.

We would like to thank the referees, who provided many useful suggestions and whose advice improved the paper. 
area have been found in connection with the Quantum Yang-Baxter Equation (QYBE), braces [44], knot theory [18], and Hopf algebras [21, 22, 8, 2]. The ring theoretical approach to QYBE has been studied for example in $[\mathbf{4 4 , ~ 1 9 , ~ 6 , ~ 5 , ~ 4 , ~ 3 , ~ 5 0 ] . ~ R e c a l l ~ t h a t ~ i n ~} 2005$ Rump introduced braces, a generalization of Jacobson radical rings, as a tool to investigate non-degenerate involutive set-theoretic solutions of the QYBE [44]. Skew braces also have been introduced recently to describe non-degenerate injective solutions of the QYBE [29]. Nil rings, and in particular nil differential polynomial rings, yield in many ways examples of braces and skew braces $[\mathbf{4 4}, \mathbf{5 0}]$. This allows to construct set-theoretic solutions of the QYBE, 1-cocycles, matched pairs of groups, exactly factorised groups, and examples of semisimple Hopf algebras by using radicals in associative noncommutative rings $[21,22,8,39,50]$.

An outline of the paper's structure now follows.

We consider properties of ideals in Ore extensions of noncommutative rings. An Ore extension of a ring $R$ is denoted by $R[X ; \sigma, \delta]$, where $\sigma$ is an endomorphism of $R$ and $\delta$ is a $\sigma$-derivation, i.e. $\delta: R \rightarrow R$ is an additive map such that $\delta(a b)=\sigma(a) \delta(b)+\delta(a) b$, for all $a, b \in R$. Recall that elements of $R[X ; \sigma, \delta]$ are polynomials in $X$ with coefficients written on the left. Multiplication in $R[X ; \sigma, \delta]$ is given by the multiplication in $R$ and the condition $X a=\sigma(a) X+\delta(a)$ for all $a \in R$.

There are two important special cases of skew polynomial rings. If $\sigma$ is the identity map, then $R[X ; \delta]$ is called a differential polynomial ring or skew polynomial ring of derivation type. If $\delta=0$, then $R[X ; \sigma]$ is called a skew polynomial ring.

Ore extensions of noncommutative rings were introduced in the 1930's by Ore. Since then they have been studied extensively. Ore extensions have been used as a source of examples of rings with various properties, and were investigated in the context of the question of which properties of rings (such as DCC or ACC on ideals and primitivity [37]) pass to differential or skew-polynomial extensions. Differential polynomial rings, an important class of Ore ring extensions, have also been investigated in relation to Lie algebras - recall that enveloping algebras of solvable Lie algebras are iterated differential polynomial rings [43, Proposition 8.3.28]. Ore ring extensions have also been studied in relation to quantum groups (see $[\mathbf{2 5}, \mathbf{1 7}])$. For detailed information about the Gelfand-Kirillov dimension of Ore extensions we refer the reader to [33]. It is also worth noticing that the conditions under which Ore extensions satisfy a polynomial identity have been completely characterized [38]. Radicals and prime ideals of differential polynomial rings over rings satisfying a polynomial identity were investigated in $[\mathbf{1 5}, \mathbf{1 2}]$. Interesting results in the 
case where $R$ is a commutative ring were obtained in $[\mathbf{2 6}, \mathbf{2 4}, \mathbf{2 7}]$; for example, in [26], the Jacobson condition (i.e., that all prime ideals are semiprimitive) is proved to pass from a commutative noetherian ring $R$ to $R[X ; \sigma, \delta]$. In $[\mathbf{2 4}]$ prime ideals in Ore extension $R[X ; \sigma, \delta]$ are investigated. In $[\mathbf{2 7}]$ Goodearl and Warfield developed necessary and sufficient conditions for a differential polynomial ring $R[X ; \delta]$ over a commutative Noetherian ring $R$ with a derivation $\delta$ to be a simple ring, a primitive ring, or a Jacobson ring.

We investigate Ore ring extensions in Sections 2, 3, 4, and 6. In Section 5 we consider twisted power series rings.

In Section 2 we investigate differential polynomial rings over rings with a non-zero Baer radical. Recall that a ring $R$ has a non-zero Baer radical if and only if it has a non-zero nilpotent ideal. It is known that, in characteristic zero, the Baer radical is stable under derivations. We show that if $R$ is a Baer radical with derivation $\delta$ then the differential polynomial ring $R[X ; \delta]$ is locally nilpotent (Theorem 2.3). This answers Question 3.2 from [41]. Some interesting related results can be found in $[35]$ and $[41]$.

In Section 3 we show that if $R=\bigoplus_{i=1}^{\infty} R_{i}$ is a graded algebra, generated in degree 1 over a field of characteristic zero, and $\delta$ is a grading preserving derivation on $R$ then $\delta(J(R)) \subseteq J(R)$, where $J(R)$ denotes the Jacobson radical of $R$ (Theorem 3.3). We show by counterexamples that the assumptions we put are necessary, and therefore this result is the "best possible".

In Section 4 we answer a question of Hong, Kim, Lee, and Nielsen from [30], in the class of algebras over infinite fields. Namely, we show that if $R$ is an algebra over an infinite field $K$ and $\delta$ is a derivation on $R$ the nil radical of $R[X ; \delta]$ equals $I[X ; \delta]$ for some ideal $I$ of $R$ (Theorem 4.2). Recall that the nil radical of a given ring is the largest nil ideal in this ring.

In [13], Bergen and Grzeszczuk consider the skew power series ring $R[[X ; \sigma, \delta]]$, and prove many interesting conjectures on such rings. They conjectured that the power series ring $R[[X ; \sigma, \delta]]$ may not be well defined, even if $\delta$ is a locally nilpotent derivation. In Section 5 we disprove their conjecture; we prove that the power series ring $R[[X ; \sigma, \delta]]$ is always well-defined, provided that $\delta$ is a locally nilpotent $\sigma$-derivation (Theorem 5.3).

In Section 6 we investigate the properties of skew polynomial rings of graded rings. Our main theorem in this section states that skew polynomial rings with natural grading are locally nilpotent if and only if they 
are graded locally nilpotent (Theorem 6.12 ). We also obtain several other results on skew polynomial rings, and pose several open questions.

All of the algebras in this paper are over a field and all rings are associative but are not required to have an identity. We denote by $A^{1}$ the usual extension with an identity of the $\operatorname{ring} A$. For a ring $R$ the Jacobson radical, upper nil radical, locally nilpotent radical, and Baer (i.e. prime) radical are denoted by $J(R), N(R), L(R), \beta(R)$, respectively. For details on general theory of radicals of rings see $[\mathbf{2 3}]$.

\section{Differential polynomial rings over rings with non-zero prime radical}

In $[41]$ we can find a diagram showing relations between radicals of a ring $R$ and radicals of the differential polynomial ring $R[X ; \delta]$. One gap visible in the diagram is the source of the wording of Question 3.2: "If $R$ is a prime radical ring with a derivation $\delta$, then is $R[X ; \delta]$ locally nilpotent (or even just Jacobson radical)?".

In this section we show that the answer to this question is positive.

Recall that a sequence $a_{0}, a_{1}, a_{2}, \ldots \in R$ where $a_{i+1} \in a_{i} R a_{i}$ (for all $i \geq$ $0)$ is called an m-sequence. An element $a$ is strongly nilpotent if each m-sequence starting with $a$ is eventually zero. The prime radical of $R$ is then precisely the set of strongly nilpotent elements in $R$. Recall that the prime radical is always locally nilpotent.

We use the following well-known graph-theoretic lemma:

Lemma 2.1 (König's lemma). Let $\mathcal{T}$ be a graph which is a tree such that every vertex has finite degree. If there exist infinitely many edges, then $\mathcal{T}$ contains an infinite path.

Let $R$ be a ring. For elements $r_{1}, r_{2}, \ldots \in R$ we denote $\beta\left(r_{1}\right)=r_{1}$, $\beta\left(r_{1}, r_{2}\right)=r_{1} r_{2} r_{1}$ and inductively we define $\beta\left(r_{1}, \ldots, r_{n+1}\right)=\beta\left(r_{1}, \ldots\right.$, $\left.r_{n}\right) r_{n+1} \beta\left(r_{1}, \ldots, r_{n}\right)$.

From Lemma 2.1 we get the following:

Corollary 2.2. Let $R$ be a Baer radical ring. Let $U_{1}, U_{2}, \ldots$ be finite subsets of $R$. Then there exists a positive integer $n$, such that for any $r_{1} \in U_{1}, r_{2} \in U_{2}, \ldots, r_{n} \in U_{n}$ we have $\beta\left(r_{1}, \ldots, r_{n}\right)=0$.

Proof: For each $r_{1} \in U_{1}$ we define the tree $T_{r_{1}}$ as follows. The vertices of $T_{r_{1}}$ are non-zero elements $\beta\left(r_{1}, t_{2}, t_{3}, \ldots, t_{m}\right)$ such that $t_{i} \in U_{i}$ for each $i$ and $m$ is a positive integer. We put an edge between vertices $\beta\left(r_{1}, t_{2}, t_{3}, \ldots, t_{m}\right)$ and $\beta\left(r_{1}, t_{2}, t_{3}, \ldots, t_{m+1}\right)$ for each $t_{i} \in U_{i}$.

Since $R$ is Baer radical, there are no infinite paths in this graph. Hence, by Lemma 2.1 there exists some positive integer $n$ such that 
every path in $T_{r_{1}}$ has length at most $n-1$. Therefore, $\beta\left(r_{1}, \ldots, r_{n}\right)=0$ for any $r_{1} \in U_{1}, r_{2} \in U_{2}, \ldots, r_{n} \in U_{n}$.

Theorem 2.3. If $R$ is a ring and $R$ is Baer (prime) radical, then for every derivation $\delta$ on $R$ the differential polynomial ring $R[X ; \delta]$ is locally nilpotent.

Proof: Let $a_{i} X^{j_{i}}$ for $i=1,2, \ldots, l$ be such that $a_{i} \in R$ and $j_{1}, \ldots, j_{l} \leq k$ for some positive integers $j_{1}, \ldots, j_{l}, k$. We will show that the subring of $R[X ; \delta]$ generated by these elements is nilpotent. Let $t$ be a positive integer number and $i_{1}, \ldots, i_{t} \leq l$. Notice that each product $\prod_{n=1}^{t} a_{i_{n}} X^{j_{i_{n}}}$ can be written as a sum of monomials of the form $a_{i_{1}}^{\left(q_{1}\right)} \cdots a_{i_{t}}^{\left(q_{t}\right)} X^{\xi}$ for some positive integers $q_{1}, \ldots, q_{t}, \xi$ where $a^{(i)}=\delta^{i}(a)$ is the $i$-th $\delta$-derivative of $a$. We say that a word (namely, a product of elements from $R$ ) $w=a_{i_{1}}^{\left(q_{1}\right)} \cdots a_{i_{t}}^{\left(q_{t}\right)}$ is good if $\left(q_{1}+1\right)+\left(q_{2}+1\right)+\cdots+\left(q_{t}+1\right) \leq t \cdot(k+1)$. Moreover, we say that $w$ has length $t$ in this presentation (notice that $t$ might differ between different presentations of $w$ ).

Part 1: We define a sequence of positive integers $p(n), f(n)$ as follows:

- Set $p(1)=1$.

- Let $f(n)$ be the number of all good words of length $p(n)$.

- Inductively define $p(n+1)$ to be the smallest number such that every good word of length $p(n+1)$ has more than $f(n)$ (separate) subwords each of which is a good word of length $p(n)$.

In this part we show by induction on $n$ that $f(n)$ is finite and that it is always possible to find $p(n+1)$ once we defined $p(1), \ldots, p(n)$. Namely, we show that $p(n), f(n)$ are well defined. For a word $v=a_{1}^{\left(m_{1}\right)} \cdots a_{t}^{\left(m_{t}\right)}$ define $\alpha(v)=\sum_{i=1}^{t}\left(m_{i}+1\right)$.

Let $w$ be a good word of length $p(n) \cdot t$ for some $t$. We can divide it into $t$ disjoint subwords $c_{1}, \ldots, c_{t}$ of length $p(n)$ each (so they have the form: $\left.c_{j}=a_{1}^{\left(m_{1}\right)} \cdots a_{p(n)}^{\left(m_{p(n)}\right)}\right)$. Since $w$ is a good word of length $p(n) \cdot t$ then $\alpha(w) \leq(k+1)(p(n) \cdot t)$. Let $y$ be the number of words $c_{i}$ such that $\alpha\left(c_{i}\right)>p(n) \cdot(k+1)$ (number of $c_{i}$ which are not good words), then $t-y$ is equal to the number of good words $c_{i}$.

It follows that $y \cdot(p(n) \cdot(k+1)+1) \leq \alpha(w) \leq p(n) \cdot t \cdot(k+1)$, therefore $y \leq \frac{p(n) \cdot t \cdot(k+1)}{p(n) \cdot(k+1)+1}$ hence $y \cdot\left(1+\frac{1}{p(n) \cdot(k+1)}\right) \leq t$ hence $t-y \geq y \cdot\left(\frac{1}{p(n) \cdot(k+1)}\right)$. Let $t>2 p(n) \cdot(k+1) \cdot f(n)$. If $y>\frac{t}{2}=p(n) \cdot(k+1) \cdot f(n)$ then $t-y \geq y \cdot\left(\frac{1}{p(n) \cdot(k+1)}\right)>f(n)$ as required (since $t-y$ is the number of good subwords in $w$ ). If $y \leq \frac{t}{2}$ then $t-y \geq \frac{t}{2}>f(n)$ as required. This shows that by taking sufficiently large $t$ we can define $p(n+1)$. It follows that $f(n+1)$ is finite. 
Part 2: Denote $U_{1}=\left\{a_{1}, a_{2}, \ldots, a_{l}\right\}$. For each $n>1$, define $U_{n}$ to be the set of (non empty) subwords of good words of length $p(n)$. By the above argument, all sets $U_{i}$ are finite.

In this part we show that every good word of length $p(n+1)$ admits $\beta\left(r_{1}, \ldots, r_{n}\right)$ as a proper subword (for some $r_{j} \in U_{j}$ ).

For $n=1$, the above assertion holds as $U_{1}=\left\{a_{1}, \ldots, a_{l}\right\}$. Suppose the assertion is valid for $n$, so every good word $w$ of length $p(n+1)$ has a proper subword $u=\beta\left(r_{1}, \ldots, r_{n}\right.$ ) (for some $r_{j} \in U_{j}$ ), namely $w=c u d$ for some (not both empty) words $c, d$.

Let $w^{\prime}$ be a good word of length $p(n+2)$. Then, by definition of $p(n+2)$ we can write $w^{\prime}=c^{\prime} w v w d^{\prime}$ where $w$ is a good word of length of $p(n+1)$, for some (possibly empty) words $c^{\prime}, d^{\prime}, v$. Hence $w^{\prime}=c^{\prime}($ cud $) v(c u d) d^{\prime}=$ $c^{\prime} c(u d v c u) d d^{\prime}$, where $c, d$ are some words and $u=\beta\left(r_{1}, \ldots, r_{n}\right)$. Denote $d v c=r_{n+1}$ and notice that $r_{n+1}$ is a subword of $w^{\prime}$, hence $r_{n+1} \in U_{n+1}$, and therefore $u d v c u=u r_{n+1} u=\beta\left(r_{1}, \ldots, r_{n+1}\right)$, as required. Note that $r_{n+1} \in R$ and $\beta\left(r_{1}, \ldots, r_{n+1}\right)$ is a proper subword of $w^{\prime}$ because $c, d$ cannot be both empty (by the inductive assumption).

Part 3: We are now ready to prove the theorem. Let $S$ be the subring of $R[X ; \delta]$ generated by elements $a_{i} X^{j_{i}}$ for $i=1, \ldots, l$ (note that every finitely generated subring of $R[X ; \delta]$ is contained in a subring generated by finitely many monomials in $X$ ).

By Corollary 2.2, there exists $n>1$ such that $\beta\left(r_{1}, \ldots, r_{n-1}\right)=0$ for all $r_{i} \in U_{i}$. Note that every element in $S^{p(n)}$ is a sum of monomials in $X$ with coefficients being good words of length $p(n)$. Every word of length $p(n)$ contains a subword $\beta\left(r_{1}, \ldots, r_{n-1}\right)=0$ for some $r_{i} \in U_{i}$. Therefore $S^{p(n)}=0$, and it follows that $R[X ; \delta]$ is locally nilpotent.

We have the following corollary from Theorem 2.3:

Corollary 2.4. Let $K$ be a field of characteristic zero and $R$ be a $K$-algebra with derivation $\delta$. Let $R[X ; \delta]$ denote the differential polynomial ring. If the Baer radical of $R$ is nonzero then the locally nilpotent radical of $R[X ; \delta]$ is nonzero.

Proof: Let $\beta(R)$ denote the Baer radical of $R$. We know by [43, Theorem 2.6.28] that $\delta(\beta(R)) \subseteq \beta(R)$, hence the differential polynomial ring $\beta(R)[X ; \delta]$ is well defined. By Theorem 2.3 we get that $\beta(R)[X ; \delta]$ is locally nilpotent. Notice that $\beta(R)[X ; \delta]$ is an ideal in $R[X, \delta]$; this concludes the proof.

\section{Stability of the Jacobson radical in graded rings}

Let $K$ be a field and let $R$ be a $K$-algebra. In [14] Bergen and Grzeszczuk have shown that nil and prime radicals need not be stable 
under a $q$-skew derivations (even in characteristic zero). On the other hand, if $K$ has characteristic 0, Proposition 2.6.28 from [43] shows that if $\delta$ is a derivation of $R$, then $\delta(N(R)) \subseteq N(R)$ and $\delta(\beta(R)) \subseteq \beta(R)$ where $N$ denotes the nil radical of $R$ and $\beta$ denotes the Baer (prime) radical of $R$. Notice that this is no longer true if $K$ is a field of characteristic $p>$ 0 . This implies that the Jacobson radical of a ring $R$ need not be $\delta$-stable, in the case when $R$ is an algebra over a field of a characteristic $p>0$.

In this section we consider the following related question:

Question 3.1. Let $R$ be an algebra over a field of characteristic zero, and let $\delta$ be a derivation on $R$. Under which assumptions is the Jacobson radical of $R \delta$-stable?

Let $R$ be a ring and let $a \in R$, we say that $I$ is the ideal generated by $a$ in $R$ if $I$ is the smallest ideal in $R$ which contains $a$. We say that a derivation $\delta$ is a grading preserving derivation on a graded ring $R=\bigoplus_{i=1}^{\infty} R_{i}$ if $\delta\left(R_{i}\right) \subseteq R_{i}$ for every $i$. For $r \in R_{i}$, denote $\operatorname{deg}(r)=i$. For an ideal $I \triangleleft R$, denote by $\delta(I)$ the ideal of $R$ generated by elements $\delta(r)$ for all $r \in I$.

Lemma 3.2. Let $R=\bigoplus_{i=1}^{\infty} R_{i}$ be an algebra over a field $K$ of characteristic zero and let $\delta$ be a grading preserving derivation on $R$. Let $I$ be a homogeneous, graded nil ideal in $R$. Then every homogeneous element from $\delta(I)$ is nilpotent.

Proof: The proof is very similar to the proof of [43, Proposition 2.6.28].

Since $I$ is homogeneous we have that $\delta(I)$ is generated by homogeneous elements of the form $\delta(a)$.

We first show that $\delta(a)$ generates a graded nil ideal for every $a \in I$ homogeneous. Let $a \in I \cap R_{j}$ for some $j$ and $c_{1}, \ldots, c_{m}, d_{1}, \ldots, d_{m} \in R^{1}$ homogeneous such that $\operatorname{deg}\left(c_{i}\right)+\operatorname{deg}(a)+\operatorname{deg}\left(d_{i}\right)=k$ for some $k$.

Since $I$ is graded nil we know that $\left(\sum_{i=1}^{m} c_{i} a d_{i}\right)^{n}=0$ for some $n$. Applying $\delta$ to this equation, we get that:

$$
0=\delta^{n}\left(\left(\sum_{i=1}^{m} c_{i} a d_{i}\right)^{n}\right)=\sum_{u} \delta^{n}\left(h_{u, 1} \cdots h_{u, n}\right),
$$

where each $h_{u, l}$ has the form $c_{i} a d_{i}$. But $\delta\left(c_{i} a d_{i}\right)=c_{i} a \delta\left(d_{i}\right)+c_{i} \delta(a) d_{i}+$ $\delta\left(c_{i}\right) a d_{i} \in c_{i} \delta(a) d_{i}+I$, so applying this to each $\delta^{n}\left(h_{u, 1} \cdots h_{u, n}\right)$ we get (iteratively using Leibniz' formula, as in [43, 1.6.29]) that:

$$
0=\delta^{n}\left(\left(\sum_{i=1}^{m} c_{i} a d_{i}\right)^{n}\right) \in m ! \cdot\left(\sum_{i=1}^{m} c_{i} \delta(a) d_{i}\right)^{n}+I .
$$


Since $m$ ! is invertible and $\sum_{i=1}^{m} c_{i} a d_{i}$ is nilpotent (being a homogeneous element from $I$ ) we get that $\sum_{i=1}^{m} c_{i} \delta(a) d_{i}$ is nilpotent, and it follows that the ideal generated by $\delta(a)$ is graded nil.

Notice that a sum of two graded nil ideals is graded nil, therefore a sum of a finite number of graded nil ideals is graded nil.

Now let $r \in \delta(I)$ be an arbitrary homogeneous element. Then $r \in$ $R^{1} \delta\left(r_{1}\right) R^{1}+\cdots+R^{1} \delta\left(r_{v}\right) R^{1}$ for some homogeneous $r_{1}, \ldots, r_{v} \in I$. Since each $R^{1} \delta\left(r_{1}\right) R^{1}$ is a homogeneous, graded nil two sided ideal, as proven above, then $R^{1} \delta\left(r_{1}\right) R^{1}+\cdots+R^{1} \delta\left(r_{v}\right) R^{1}$ is graded nil too, and so $r$ is nilpotent and the result follows.

We are ready to prove our next result. By $M_{k}(R)$ we will denote the $k$-by- $k$ matrix ring with entries from $R$.

Theorem 3.3. Let $R=\bigoplus_{i=1}^{\infty} R_{i}$ be an algebra over a field $K$ of characteristic zero, and suppose that $R$ is generated in degree one $\left(R_{1}\right.$ generates $R$ as an algebra). If $\delta$ is a grading preserving derivation on $R$ then $\delta(J(R)) \subseteq J(R)$.

Proof: Let $a \in J(R)$; we need to show that $\delta(a) \in J(R)$. By a result of Bergman [16] the Jacobson radical of an $\mathbb{N}$-graded ring is homogeneous, so we only need to consider the case when $a$ is homogeneous. Let $I$ be the ideal generated by $a$ in $R$. Notice that $I$ is a graded Jacobson radical ring (as the quasi-inverse of any element from $I$ is in $I$ and $I$ is homogeneous).

Notice that for every $k$ the matrix ring $M_{k}(R)$ has natural grading inherited from $R$ as $M_{k}(R)=\bigoplus_{i=1}^{\infty} M_{k}\left(R_{i}\right)$. It is known that matrix rings over Jacobson radical rings are Jacobson radical hence for every $k$, the matrix ring $M_{k}(I)$ is Jacobson radical. Every graded Jacobson radical ring is graded nil, hence $M_{k}(I)$ is a graded nil ideal in $M_{k}(R)$. Notice that $\delta$ is a grading preserving derivation on $M_{k}(R)$ (where the derivation of a matrix with entries $a_{i, j}$ is the matrix with entries $\left.\delta\left(a_{i, j}\right)\right)$. Let $\tilde{I}$ be the ideal of $R$ generated by $\delta(a)$. By Lemma 3.2 every homogeneous matrix from $M_{k}(\tilde{I})$ is nilpotent.

Let $c \in \tilde{I}$, then by Definition 7.1 from [48] there is a matrix $X$ with entries from $R_{1}$ such that $X^{n}=0$ for some $n$ if and only if $c$ is quasiinvertible (see Lemma 7.2 in [48]). Moreover, by Lemma 7.1 from [48] it follows that there is $m$ such that all entries of $X^{m}$ are in the ideal generated by the homogeneous components of $c$. In our situation, we get that there is $m$ such that all entries of $X^{m}$ are in the ideal generated by $\delta(a)$. By the above $X^{n}=0$ for some $n$, and hence $c$ is quasi-invertible. It follows that every element from $\tilde{I}$ is quasi-invertible and hence it is in the Jacobson radical of $R$. This concludes the proof. 
Before we end this section, let us provide two examples which demonstrate the sharpness of Theorem 3.3. Namely, neither one of the assumptions in the formulation of Theorem 3.3 can be dropped.

We begin by exhibiting a graded algebra which is generated in degree 1, such that the Jacobson radical is not stable under some grading preserving derivation. This marks the necessity of the assumption in Theorem 3.3 that the characteristic of the base field is zero.

Example 3.4 (The characteristic hypothesis is necessary). Let $F$ be an arbitrary field of characteristic $p>0$. Let $R=F[x, y] /\left\langle x^{p}\right\rangle$. Then $R$ is graded by $\operatorname{deg}(x)=0, \operatorname{deg}(y)=1$. Consider the (non-unital) subalgebra $R_{+}=F\left[y, x y, \ldots, x^{p-1} y\right]$. Note that $R_{+}$is a graded algebra which is generated in degree 1 .

Define a derivation $\delta: R_{+} \rightarrow R_{+}$by $\delta(y)=0, \delta(x y)=y, \ldots, \delta\left(x^{p-1} y\right)=$ $(p-1) y x^{p-2}$. Note that $\delta$ preserves the grading on $R_{+}$. Observe that $x y \in J\left(R_{+}\right)$but $\delta(x y)=y \notin J\left(R_{+}\right)$.

We now show that the grading assumption cannot be dropped, even when the characteristic is zero. Namely, we construct an algebra over a field of characteristic zero, with a derivation such that the Jacobson radical is not stable under it. This shows that the grading hypothesis cannot be dropped. Since we can always artificially declare that the whole algebra has degree zero, it shows that an additional assumption on the grading must be put to prevent such redundant situations - in our case, we assume the algebra is generated in degree 1 .

Example 3.5 (The grading hypothesis is necessary). Recall the examples mentioned in [14] in positive characteristic: for any prime $p$ let $R_{p}=\mathbb{F}_{p}[x] /\left\langle x^{p}\right\rangle$ with derivation $\delta_{p}: R_{p} \rightarrow R_{p}$ defined by $\delta_{p}(x)=1$.

Now let $\mathcal{P}=\{2,3,5, \ldots\}$ be the set of prime numbers. Fix a non-principal ultrafilter $\mathcal{F}$ on $\mathcal{P}$ and let $\hat{R}=\prod_{\mathcal{F}} R_{p}$ be the corresponding ultraproduct. Note that the set of derivations $\left\{\delta_{p}\right\}_{p \in \mathcal{P}}$ can be glued to a derivation $\hat{\delta}: \hat{R} \rightarrow \hat{R}$.

Note that $\hat{R}$ is an algebra over $\prod_{\mathcal{F}} \mathbb{F}_{p}$, which is a field of characteristic zero. Observe that the diagonal element $\hat{x}=(x, x, \ldots)$ is contained in the Jacobson radical of $\hat{R}$. To see this, observe that the natural lift of $\hat{x}$ to the standard product $\prod_{p \in \mathcal{P}} R_{p}$ generates a quasi-invertible ideal, and hence also its image (i.e. $\hat{x})$ does in $\hat{R}$. However, $\hat{\delta}(\hat{x})=1 \notin J(\hat{R})$.

Question 3.6. Let $R$ be a graded algebra of characteristic zero and $\delta$ be a grading preserving derivation on $R$. Is the Brown-McCoy radical $\delta$-stable? Is the Behrens radical $\delta$-stable? 


\section{On the nil radical of differential polynomial rings}

In [30], Hong, Kim, Lee, and Nielsen conjectured that for any ring $R$ and a derivation $\delta$ of $R$

$$
N(R[X ; \delta])=I[X ; \delta]
$$

for some ideal $I$ in $R$. We prove that this conjecture holds for algebras over infinite fields. Namely, we show that the nil radical of a differential polynomial ring $R[X ; \delta]$ equals $I[X ; \delta]$ for some ideal $I$ in $R$, provided that $R$ is an algebra over an infinite field. Since we use classical methods developed by Amitsur and Jacobson the content of this section can be viewed as a folklore; we provide detailed proofs for the convenience of the reader.

Lemma 4.1. Let $R$ be an algebra over an infinite field $K$ and let $\delta$ be a derivation on $R$. Suppose that the nil radical of $R[X ; \delta]$ is non-zero. Then there is $0 \neq a \in R$ which is in the nil radical of $R[X ; \delta]$.

Proof: Let $f=f(X) \in R[X ; \delta]$ be a non-zero polynomial of the minimal possible degree and such that $f \in N(R[X ; \delta])$. If the degree of $f$ is 0 then $f \in R$ and the result follows. Suppose now on the contrary that the degree of $f(X)$ is bigger than 0 . For arbitrary $t \in K$ denote $g_{t}(X)=$ $f(X+t)-f(X)$. Notice that the degree of $g_{t}(X)$ is smaller than the degree of $f(X)$. We will show that $g_{t}(X)$ is in the nil radical of $R[X ; \delta]$. It suffices to show that $f(X+t)$ is in the nil radical of $R[X ; \delta]$. To get this fact, notice that the mapping $\alpha_{t}: R[X ; \delta] \rightarrow R[X ; \delta]$ given by $\alpha(r)=r$ for $r \in R$ and $\alpha(X)=X+t$ is a homomorphism of rings since $\alpha_{t}(r X-$ $X r+\delta(r))=r(X+t)-(X+t) r+\delta(r)=0$ for every $r \in R$. In fact, this is easy to see that $\alpha$ is even an isomorphism of rings. Now, obviously we have $f(X+t)=\alpha_{t}(f(X)) \in N(R[X ; \delta])$, as $f(X) \in N(R[X ; \delta])$. Thus $g_{t}(X) \in N(R[X ; \delta])$.

Since $K$ is an infinite field, there exists some $t$ such that $\alpha_{t}(X)=$ $f(X+t)-f(X) \neq 0$, this implies that $g_{t}(X)=f(X+t)-f(X)$ is a nonzero element of a smaller degree that $f(X)$ and $g_{t}(X) \in N(R[X ; D])$. We have obtained a contradiction, since we assumed that the degree of $f(X)$ is the minimal possible.

Theorem 4.2. Let $R$ be an algebra over an infinite field $K$ and let $\delta$ be a derivation on $R$. Then the nil radical of $R[X ; \delta]$ equals $I[X ; \delta]$ for some ideal $I$ of $R$.

Proof: It follows from [30, Proposition 4.3] and from the above Lemma 4.1.

Notice that our proof bears similarity to the original approach used by Amitsur to show that the Jacobson radical of a polynomial ring $R[X]$ 
equals $I[X]$ for some ideal $I$ of $R$. This approach is well described and applied in [30, Section 5], wherein it is shown that the nil radical of a differential polynomial ring $R[X ; \delta]$ has a non-zero intersection with $R$, provided that $R$ is an algebra over a field of characteristic zero.

Remark 4.3. Notice that by using a similar proof as in Lemma 4.1 it can be shown that the Behrens and Brown-McCoy radical of a differential polynomial ring $R[X ; \delta]$ is of the form $I[X ; \delta]$ for some ideal $I$ of $R$, provided that $R$ is an algebra over an infinite field.

Remark 4.4. Note that if $I \triangleleft R[X]$ is a non-zero $\sigma_{t}$-invariant ideal $\left(\sigma_{t}(X)=\right.$ $X+t$ for $t$ scalar) then $I \cap R \neq 0$. Therefore $P(R[X])=(P(R[X]) \cap R)[X]$ for any radical class $P$ of algebras over infinite fields. See also [34, Exercise 5.3]. Similarly, using the automorphisms $\gamma_{t}(X)=t X$ one can show that $P(R[X ; \sigma])$ is homogeneous (and if $\sigma$ is an automorphism, then also $P\left[X, X^{-1} ; s\right]$ is).

This remark was pointed out to us by the referee, to whom we thank for that.

\section{Skew power series rings}

Consider a ring $R$ with an endomorphism $\sigma$. Recall that, given an endomorphism $\sigma$ of a ring $R$, a derivation $\delta$ of $R$ is called a $\sigma$-derivation if $\delta(x y)=\delta(x) y+\sigma(x) \delta(y)$.

In [13], the skew power series ring $R[[X ; \sigma, \delta]]$ is considered, and many interesting results are proved on such rings. The authors conjecture that '... even if $\delta$ is locally nilpotent, when we drop the assumption that $\sigma=1$, another problem can arise [...] note that even if $\delta$ is locally nilpotent, this sum might not be defined in $R$ '.

Our aim in what follows is to show that, in contrast with the quoted remark, the ring $R[[X ; \sigma, \delta]]$ always has well-defined multiplication, extending that of $R[X ; \sigma, \delta]$, provided that $\delta$ is a locally nilpotent derivation. This opens a gateway for many possible research problems as we propose in the end, as well as for generalizations of results previously proven only when additional restrictions on $\sigma, \delta$ are put.

For $w=w(\delta, \sigma)$ a monomial in $\sigma, \delta$, write $w(a)$ for evaluation of $w$ at $a$ (for $a \in R$ ), and by $\operatorname{deg}_{\sigma}(w), \operatorname{deg}_{\delta}(w)$ denote the number of occurrences of $\sigma, \delta$ respectively in $w$.

Lemma 5.1. Let the notation be as above, and let $a \in R$. The formula:

$$
X^{n} a=\sum_{m=0}^{n}\left(\sum_{\substack{\operatorname{deg}_{\sigma}(w)=m \\ \operatorname{leg}_{\delta}(w)=n-m}} w(\delta, \sigma)(a)\right) X^{m}
$$

holds in $R[X ; \sigma, \delta]$. 
Proof: By induction. The case $n=1$ follows immediately, since $X a=$ $\sigma(a) X+\delta(a)$ by definition. Now assume validity for $n$, and consider

$$
X^{n+1} a=\sum_{m=0}^{n}\left(\sum_{\substack{\operatorname{deg}_{\sigma}(w)=m \\ \operatorname{deg}_{\delta}(w)=n-m}} X w(\delta, \sigma)(a)\right) X^{m}
$$

which decomposes to a sum of the action of $\sigma$ and action of $\delta$ on coefficients:

$$
\sum_{m=0}^{n}\left(\sum_{\substack{\operatorname{deg}_{\sigma}(w)=m \\ \operatorname{leg}_{\delta}(w)=n-m}} \sigma w(a)\right) X^{m+1}+\sum_{m=0}^{n}\left(\sum_{\substack{\operatorname{deg}_{\sigma}(w)=m \\ \operatorname{leg}_{\delta}(w)=n-m}} \delta w(a)\right) X^{m}
$$

collecting monomials of equal degrees we obtain:

$$
\sum_{m=0}^{n+1}\left(\sum_{\substack{\operatorname{deg}_{\sigma}(w)=m \\ \operatorname{deg}_{\delta}(w)=n+1-m}} w(\delta, \sigma)(a)\right) X^{m}
$$

as desired.

For $f \in R[X ; \sigma, \delta]$, we denote by $(f)_{m}$ the coefficient of its degree $m$ component.

Lemma 5.2. Fix $a \in R$ and assume $\delta$ is locally nilpotent. Then for every $m$ there exists $N_{m}=N_{m}(a)$ such that for all $n \geq N_{m}$ and all $i \leq m$ we have:

$$
\left(X^{n} a\right)_{i}=0
$$

Proof: By induction. The case $m=0$ is done by taking $N_{0}$ such that $\delta^{N_{0}}(a)=0$ (which exists, by assumption that $\delta$ is locally nilpotent).

We now suppose that we have defined all $N_{m}$ and we define $N_{m+1}$. By Lemma 5.1, for all $i \leq m$,

$$
0=\left(X^{N_{m}} a\right)_{i}=\sum_{\substack{\operatorname{deg}_{\sigma}(w)=i \\ \operatorname{deg}_{\delta}(w)=N_{m}-i}} w(a) .
$$

Denote $\operatorname{deg}(w)=\operatorname{deg}_{\sigma}(w)+\operatorname{deg}_{\delta}(w)$. Consider all monomials $w=$ $w(\delta, \sigma)$ with $\operatorname{deg}(w)=N_{m}$, and let $l$ be large enough such that $\delta^{l}(w(a))=$ 0 for all such monomials. Finally, take $N_{m+1}=l+N_{m}$. Observe that for all $i \leq m$ we have $\left(X^{N_{m+1}} a\right)_{i}=0$, since $N_{m+1}>N_{m}$. We need to show that $\left(X^{N_{m+1}} a\right)_{m+1}=0$. 
Given a monomial $w=w(\delta, \sigma)$ with $\operatorname{deg}(w)=N_{m+1}$, write $w=w_{1} w_{2}$ where $\operatorname{deg}\left(w_{1}\right)=l$ and $\operatorname{deg}\left(w_{2}\right)=N_{m}$. Recall that by Lemma 5.1

$$
\left(X^{N_{m}} a\right)_{m+1}=\sum_{\substack{\operatorname{deg}_{\sigma}(w)=m+1 \\ \operatorname{deg}_{\delta}(w)=N_{m+1}-m+1}} w(a) .
$$

By (1), it follows that either all $m+1$ occurrences of $\sigma$ are in $w_{2}$ or $w_{2}(a)=0$ (and hence $w(a)=0$ ). Therefore $w_{1}=\delta^{l}$, and by definition of $l$ we now have that $w(a)=0$.

Theorem 5.3. Let $R$ be an algebra, $\sigma$ an endomorphism, and $\delta$ a $\sigma$-derivation which is locally nilpotent. Then natural extension of the multiplication from $R[X ; \sigma, \delta]$ to $R[[X ; \sigma, \delta]]$ is well defined.

Proof: Let $f=\sum_{i=0}^{\infty} c_{i} X^{i}$ and $f^{\prime}=\sum_{i=0}^{\infty} c_{i}^{\prime} X^{i}$. Then we need to show that we can calculate $\left(f f^{\prime}\right)_{m}$ for every $m \geq 0$. Fix such $m$. Let $q=$ $\max _{0 \leq i \leq m}\left\{N_{m}\left(c_{i}^{\prime}\right)\right\}$. Then:

$\left(f f^{\prime}\right)_{m}=\left(\left(\sum_{i=0}^{\infty} c_{i} X^{i}\right) \cdot\left(\sum_{i=0}^{m} c_{i}^{\prime} X^{i}\right)\right)_{m}=\left(\left(\sum_{i=0}^{q} c_{i} X^{i}\right) \cdot\left(\sum_{i=0}^{m} c_{i}^{\prime} X^{i}\right)\right)_{m}$

which is a well defined element of $R$.

This opens several interesting research directions; in $[\mathbf{1 2}]$ it is proved that if $R$ is locally nilpotent PI then $R[X ; \delta]$ is Jacobson radical.

Question 5.4. Can one provide a good characterization of the Jacobson radical of $R[[X ; \sigma, \delta]]$ in terms of $R$ and $\sigma, \delta$ ? When is it the case that $R[[X ; \sigma, \delta]]$ is Jacobson radical? When is it semiprimitive?

\section{Graded algebras, homogeneity, and some related questions}

Recall that a ring is said to be Brown-McCoy radical if it cannot be mapped onto a ring with 1, and Behrens radical if it cannot be mapped onto a ring with a non-zero idempotent. Recall also that a graded ring is graded nil if all homogeneous elements in $R$ are nilpotent. A number of papers regarding radical properties of graded nil rings have been published. It was proven in [42] that if $R$ is nil then $R[X]$ is BrownMcCoy radical, and later, in [9], it was shown that $R[X]$ is Behrens radical. Recently, it was proved that for any nil ring $R$ the polynomial ring $R\left[X_{1}, \ldots, X_{n}\right]$ with arbitrarily many variables is Brown-McCoy radical (see $[\mathbf{2 0}]$ ).

While an arbitrary $\mathbb{Z}$-graded ring which is graded nil is Brown-McCoy radical, for any $\mathbb{Z}$-graded nil ring $R$ of characteristic $p>0$ the polynomial 
ring $R[X]$ is Brown-McCoy radical (see $[\mathbf{3 6}]$ ). It is not known if the latter result holds for algebras over fields of arbitrary characteristic.

Proposition 6.1. Let $R=\bigoplus_{i=0}^{\infty} R_{i}$ be graded nil. Then $R$ is Behrens radical.

Proof: First, observe that we may assume that $R$ is positively graded, namely that $R_{0}=0$. To see this, let $\varphi: R \rightarrow P$ be a surjective homomorphism, where $0 \neq e \in P$ is an idempotent. Pick a lift of $e$, say $x=x_{0}+x_{1}+\cdots+x_{n}$, where $x_{i} \in R_{i}$. Since $R$ is graded nil, there exists some exponent $m$ such that $x_{0}^{m}=0$, hence $x^{m} \in \bigoplus_{i=1}^{\infty} R_{i}$. But $\varphi\left(x^{m}\right)=e^{m}=e$.

Hence we assume that $R$ is positively graded. By Zorn's lemma, there exists an ideal $I \triangleleft P$ maximal with respect to not containing $e$. We replace $P$ by $P / I$. Note that $P$ must be primitive. First, it is semiprimitive (since Jacobson radicals cannot contain non-zero idempotents). Then it follows that the zero ideal is an intersection of primitive ideals. Since $e \neq 0$, it follows that at least one of them is zero, hence $P$ is a primitive ring. By $[47]$ we have that $P$, as a primitive quotient of a (positively) graded nil ring, is graded too. Write $e=a_{1}+\cdots+a_{r}$ with $a_{i}$ homogenous of degree $i$ and $r$ maximal such that $a_{r} \neq 0$. Then $\psi(e)=\psi\left(e^{r+1}\right)$ is supported on the homogeneous components of degrees $r+1, r+2, \ldots$, a contradiction.

Remark 6.2. It can be shown that primitive ideals in non-negatively graded nil rings are homogeneous, applying modified arguments from $[47]$ (however we have chosen not to include a full proof here).

There is a natural question which arises in the light of both Proposition 6.1 and $[\mathbf{3 6}]$ :

Question 6.3. Is a $\mathbb{Z}$-graded nil ring Behrens radical? What if we assume, in addition, that the characteristic is $p>0$ ?

It is known that the Jacobson radical of a $\mathbb{Z}$-graded ring is homogeneous [16]; similarly, it was shown that the Brown-McCoy radical of a $\mathbb{Z}$-graded ring is homogeneous $[\mathbf{3 1}]$. The following question then naturally arises:

Question 6.4. Is the Behrens radical of a $\mathbb{Z}$-graded ring homogeneous?

We also ask the following:

Question 6.5. Is there a locally nilpotent ring $R$ and a derivation $\delta$ such that $R[X ; \delta]$ maps onto a ring with a non-zero idempotent? 
Clearly, if $R$ is locally nilpotent then so are $R[X]$ and $R\left[X, X^{-1}\right]$. By [32], idempotents in $R\left[X, X^{-1}\right]$ arise from idempotents in $R$, hence for $R$ without non-zero idempotents (e.g. $R$ nil) also $R\left[X, X^{-1}\right]$ has no non-zero idempotents. This is done by embedding the Laurent extension $R\left[X, X^{-1}\right]$ into $R[[X]]$. This embedding does not seem to be valid for twisted Laurent extensions, and we ask:

Question 6.6. Suppose $R$ is a ring with an automorphism $\sigma$ and a $\sigma$-derivation $\delta$. Is it possible that $R$ admits no non-zero idempotents but $R\left[X, X^{-1} ; \sigma, \delta\right]$, the left localization of $R[X ; \sigma, \delta]-$ whenever it exists, does? What if $R$ is locally nilpotent? What if $\delta=0$ ?

Observe that every ring with an identity is an idempotent ring, i.e. $R^{2}=R$ (where $R^{2}$ consists of a finite sum of products of at least two elements from $R$ ). It is known that every finitely generated idempotent ring gives rise to a perfect group [40]. For this reason, idempotent rings are related to several important open questions in group theory [40]. We ask:

Question 6.7. What can be said about idempotent rings $R=R^{2}$ which are Brown-McCoy radicals? Behrens radicals?

Question 6.8. What can be said about rings which cannot be homomorphically mapped onto an idempotent ring, i.e. onto a ring $R$ such that $R^{2}=R$ ? Would such property give rise to a radical property?

Notice that, by Nakayama's lemma, a finitely generated Jacobson radical ring cannot be homomorphically mapped onto an idempotent ring.

Question 6.9. What can be said about rings which cannot be homomorphically mapped onto a ring with a non-zero idempotent subring?

Remark 6.10. Note that there exists a finitely generated Jacobson radical algebra which contains a non-zero idempotent subring. Indeed, by Theorem 4.1 from [1] it is possible to embed an arbitrary countable dimensional Jacobson radical algebra inside a finitely generated Jacobson radical algebra. Since countable dimensional Jacobson radical idempotent rings exist (e.g. see [45]), it proves the statement.

The ring $R[X ; \sigma]$ is always graded by $\operatorname{deg}(r)=0, \operatorname{deg}\left(r X^{j}\right)=j$ (for all non-zero $r \in R$ and all natural numbers $j>0$ ). Therefore, if $R[X ; \sigma]$ is Jacobson radical then it is graded nil (and in particular $R$ is nil). We will use this grading in all of the following results in this section.

In [7, Theorem 3.8], it is shown that over an uncountable field, $R[X ; \sigma]$ is nil if $R$ is nil and $\sigma$ is locally torsion. Recall that $\sigma$ is locally torsion 
if, for every $r \in R$, there is $n=n(r)$ such that $\sigma^{n}(r)=r$. We note that, for general base fields, we have a weaker result.

Remark 6.11. If $R$ is nil and $\sigma$ is locally torsion, then $R[X ; \sigma]$ is graded nil.

Proof: Let $r \in R$; we want to show that $r X^{d}$ is nilpotent. Let $t$ be the order of $\sigma$ on $r$, and let $n$ be the nilpotency index of $r \sigma^{d}(r) \cdots \sigma^{d(t-1)}(r)$. Then

$$
\begin{aligned}
& \left(r X^{d}\right)^{t n}=\left(r \sigma^{d}(r) \cdots \sigma^{d(t-1)}(r) X^{d t}\right)^{n} \\
& \quad=\left(r \sigma^{d}(r) \cdots \sigma^{d(t-1)}(r)\right)^{n} X^{d t n}=0 .
\end{aligned}
$$

Motivated by the questions posed in [28], in [11], finitely generated infinite dimensional graded nilpotent, primitive algebras were constructed. Later, in [10], a graded nilpotent algebra containing a free subalgebra was constructed. In particular, graded nilpotent algebras need not be nil.

Our Theorem 6.12 shows that this phenomenon is impossible in the context of $R[X ; \sigma]$.

A graded algebra is graded nilpotent if the algebra generated by any set of homogeneous elements of the same degree is nilpotent.

It is graded locally nilpotent if the algebra generated by any finite set of homogeneous elements of the same degree is nilpotent.

We prove the following.

Theorem 6.12. Suppose $R[X ; \sigma]$ is graded locally nilpotent. Then it is locally nilpotent.

We begin with the following lemma:

Lemma 6.13. Let $f=r_{1} X^{i_{1}} \cdots r_{n} X^{i_{n}} \in R[X ; \sigma]$ be a product of homogeneous elements of degrees $i_{1}, \ldots, i_{n}$ (all $r_{i}$ 's are from $R$ ). Suppose $m \geq i_{1}, \ldots, i_{n}$. Then $f$ can be written as

$$
f=s_{1} X^{m} \cdots s_{l} X^{m} s_{l+1} X^{m^{\prime}},
$$

where $m^{\prime} \leq 2 m$ and $s_{1}, \ldots, s_{l+1} \in R$. Moreover, $l m+m^{\prime}=i_{1}+\cdots+i_{n}$.

Proof: This is done by induction on $n$. The case $n=1$ is clear, since $f=r_{1} X^{i_{1}}$, and we can take $l=0$ and $m^{\prime}=i_{1} \leq m$.

Now consider a product of $n+1$ homogeneous elements,

$$
f=r_{1} X^{i_{1}} \cdots r_{n+1} X^{i_{n+1}} .
$$

By induction, we can write

$$
f=s_{1} X^{m} \cdots s_{l} X^{m} s_{l+1} X^{m^{\prime}} r_{n+1} X^{i_{n+1}},
$$

with $m^{\prime} \leq 2 m$. 
If $m^{\prime}=m$ we are done, since $i_{n+1} \leq m$. If $m^{\prime}>m$, write $m^{\prime}=m+d$ (with $d \leq m$ ). Now

$$
\begin{aligned}
f & =s_{1} X^{m} \cdots s_{l} X^{m} s_{l+1} X^{m^{\prime}} r_{n+1} X^{i_{n+1}} \\
& =s_{1} X^{m} \cdots s_{l} X^{m} s_{l+1} X^{m} \sigma^{d}\left(r_{n+1}\right) X^{d+i_{n+1}}
\end{aligned}
$$

and we are done, since $d, i_{n+1} \leq m$ and therefore $d+i_{n+1} \leq 2 m$.

Otherwise, $m^{\prime}<m$, so $m^{\prime}+i_{n+1} \leq 2 m$ and

$$
\begin{aligned}
f & =s_{1} X^{m} \cdots s_{l} X^{m} s_{l+1} X^{m^{\prime}} r_{n+1} X^{i_{n+1}} \\
& =s_{1} X^{m} \cdots s_{l} X^{m} s_{l+1} \sigma^{m^{\prime}}\left(r_{n+1}\right) X^{m^{\prime}+i_{n+1}}
\end{aligned}
$$

and we are done.

The equality $l m+m^{\prime}=i_{1}+\cdots+i_{n}$ follows from comparing the degrees of both sides.

We are now ready to prove Theorem 6.12 .

Proof of Theorem 6.12: Pick $f_{1}, \ldots, f_{n} \in R[X ; \sigma]$ where $f_{i}=\sum_{j=0}^{m_{i}} r_{i, j} X^{j}$. Set $m=\max _{i} m_{i}$. Let $S=\left\{\sigma^{k}\left(r_{i, j}\right) \mid 1 \leq i \leq n, 1 \leq j \leq m_{i}, 0 \leq k \leq\right.$ $m$ \} (where $\sigma^{0}=\mathrm{id}$ ) and let $T$ be the set of all products of up to $m$ (not necessarily distinct) elements from $S$, namely $T=\bigcup_{i=1}^{m} S^{i}$. Let $U=\left\{t X^{m} \mid t \in T\right\}$. Since $R[X ; \sigma]$ is graded locally nilpotent, and $U$ is a set of homogeneous elements of degree $m$, there is some $d$ such that $U^{d}=0$. Since $R$ is locally nilpotent, we have that for some exponent $e$ the set of all products of $e$ zero-degree coefficients vanish, namely $\left\{r_{1,0}, \ldots, r_{n, 0}\right\}^{e}=0$.

Let $N=(d+2) m e$. Then for every choice of indices $i_{1}, \ldots, i_{N} \in$ $\{1, \ldots, n\}$, we can write $f_{i_{1}} \cdots f_{i_{N}}=g+h$, where $g$ is a sum of homogeneous elements of degrees not exceeding $(d+2) m-1$, and $h$ is a sum of homogeneous elements of degrees at least $(d+2) m$.

First, we claim that $g=0$. Indeed, given a product of $N=(d+2) m e$ elements of the form $r_{i, j} X^{j}$, say, $r_{i_{1}, j_{1}} X^{j_{1}} \cdots r_{i_{N}, j_{N}} X^{j_{N}}$ of degree less than $(d+2) m$, there must be some $1 \leq v \leq N-e+1$ such that $j_{i_{v}}=\cdots=j_{i_{v+e-1}}=0$, because otherwise it would have degree at least $(d+2) m$. It follows that

$$
r_{i_{1}, j_{1}} X^{j_{1}} \cdots r_{i_{N}, j_{N}} X^{j_{N}}=\alpha \cdot r_{i_{v}, 0} \cdots r_{i_{v+e-1}, 0} \cdot \beta=0 .
$$

Finally, we claim that $h=0$. Indeed, take a product $r_{i_{1}, j_{1}} X^{j_{1}} \ldots$ $r_{i_{N}, j_{N}} X^{j_{N}}$ of degree at least $(d+2) m$. By Lemma 6.13 , we can present it as a product $s_{1} X^{m} \cdots s_{l} X^{m} s_{l+1} X^{m^{\prime}}$, where $m^{\prime} \leq 2 m$ and $l m+m^{\prime}=$ $i_{1}+\cdots+i_{N} \geq(d+2) m$. It follows that $(l+2) m \geq l m+m^{\prime} \geq(d+2) m$, so $l \geq d$. Since $U^{d}=0$ and each $s_{i} X^{m} \in U$, we have that $s_{1} X^{m} \cdots s_{l} X^{m}=0$; this concludes the proof. 
For a graded ring $S$, we denote by $S^{+}$the positive part; i.e. if $S=$ $R[X ; \sigma]$ then $S^{+}=S X+S X^{2}+\cdots$

It is sometimes a useful tool to pass to the skew power series ring $R[[X ; \sigma]]$ to prove results on $R[X ; \sigma]$ :

Proposition 6.14. Let $R$ be an algebra with an endomorphism $\sigma$. Let $J(R[X ; \sigma])$ denote the Jacobson radical of the skew polynomial ring $R[X, \sigma]$. Let $I=R \cap J(R[X ; \sigma])$. Then $I[X ; \sigma]^{+}$is graded nil. If $R$ contains an uncountable field then $J(R[X ; \sigma])^{+}$is nil.

Note that in general graded nil does not imply nil, even over uncountable fields.

Proof: Pick $c \in I$ and $p \in \mathbb{N}$. Compute in $R[[X ; \sigma]]$ :

$$
\left(1-c X^{p}\right)^{-1}=1+c X^{p}+c \sigma^{p}(c) X^{2 p}+c \sigma^{p}(c) \sigma^{2 p}(c) X^{3 p}+\cdots
$$

On the other hand, we know that in $R[X ; \sigma]$ there is a finite polynomial representing $\left(1-c X^{p}\right)^{-1}$, so they must coincide. Hence for $m \gg 1$ we have $\prod_{i=0}^{m} \sigma^{i p}(c)=0$, hence $\left(c X^{p}\right)^{m}=0$.

Now assume $R$ contains an uncountable field, $k$. Pick $f \in J(R[X ; \sigma])^{+}$ and consider $g=(1-\alpha f)^{-1}-1$; then there exists $n$ such that for infinitely many such $\alpha$ 's we have that $g \in R X+R X^{2}+\cdots+R X^{n}$. On the other hand, in $R[[X ; \sigma]]$ we have that $g=\alpha f+\alpha^{2} f^{2}+\cdots$, so $(g)_{m}=\alpha(f)_{m}+\alpha^{2}\left(f^{2}\right)_{m}+\cdots+\alpha^{m}\left(f^{m}\right)_{m}$ (since $f^{m+1}, f^{m+2}, \ldots$ have zero coefficient of $\left.X^{m}\right)$. For all $m \geq n+1$, we see that $(g)_{m}=0$, so the invertible Vandermonde matrix $\left(\alpha_{i}\right.$ are chosen to be non-zero distinct scalars)

$$
\left(\begin{array}{cccc}
1 & \alpha_{1} & \ldots & \alpha_{1}^{m-1} \\
1 & \alpha_{2} & \ldots & \alpha_{2}^{m-1} \\
\vdots & \vdots & \ddots & \vdots \\
1 & \alpha_{m} & \ldots & \alpha_{m}^{m-1}
\end{array}\right)
$$

annihilates the vector $\left((f)_{m}\left(f^{2}\right)_{m} \cdots\left(f^{m}\right)_{m}\right)^{T}$, hence $\left(f^{i}\right)_{m}=0$ for all $i \in \mathbb{N}$. We get that on the one hand all coefficients of $X^{j}$ in $f^{n+1}$ are zero for $j \leq n$, on the other hand as we have shown $\left(f^{i}\right)_{m}=0$ for all $m \geq n+1$, so we conclude that $f^{n+1}=0$, and so $J(R[X ; \sigma])^{+}$is nil.

Corollary 6.15. If $R$ is a nil algebra over an uncountable field, then $J(R[X ; \sigma])$ is nil.

Proof: Pick $x \in J(R[X ; \sigma])$ and denote by $r \in R$ its constant term. Then $r$ is nilpotent, say $r^{n}=0$, so $x^{n}=(r+(x-r))^{n} \in J(R[X ; \sigma])^{+}$; hence by Proposition 6.14 it is nilpotent, and we are done. 
We find the following question interesting:

Question 6.16. Suppose $R[X ; \sigma]$ is graded nil (or Jacobson radical), and $R$ is locally nilpotent. Does it follow that $R[X ; \sigma]$ is nil (or Jacobson radical)?

Note that the question is closely related to the Köthe problem: for $\sigma=$ $\mathrm{id}$, the assertion that $R$ is nil implies that $R[X ; \sigma]=R[X]$ is Jacobson radical is equivalent to an affirmative answer to the Köthe problem. Note that if we assume $R$ is nil, but not necessarily locally nilpotent, then $R[X]$ need not be nil $[\mathbf{4 6}]$.

By [51], if $R$ is an algebra over an uncountable field and $\delta$ is locally nilpotent, then $J(R[X ; \delta]) \cap R$ is nil. Note that if $\delta$ is replaced with an endomorphism $\sigma$ then it is still true (even over an arbitrary base field), since the resulting set is homogeneous and the Jacobson radical of a $\mathbb{Z}$-graded algebra is graded nil.

Question 6.17. Consider $J(R[X ; \sigma, \delta]) \cap R$. Is it nil if we assume that $\delta$ is locally nilpotent? What if we assume that $\sigma$ is locally torsion?

Notice that it was shown in $[\mathbf{4 9}]$ that $J(R[X ; \delta]) \cap R$ is nil provided that $\delta$ is a locally nilpotent derivation and $R$ is an algebra over a field of characteristic $p>0$. The assumption that $\delta$ is a locally nilpotent derivation is necessary (see [49, Theorem 1]). It is not known if the assumption that the base field has a non-zero characteristic is necessary.

\section{References}

[1] A. N. Alahmadi, H. H.Alsulami, S. K. Jain, and E. I. Zelmanov, Matrix wreath products of algebras and embedding theorems, Trans. Amer. Math. Soc. (to appear).

[2] I. Angiono, C. Galindo, and L. Vendramin, Hopf braces and Yang-Baxter operators, Proc. Amer. Math. Soc. 145(5) (2017), 1981-1995. DOI: 10.1090/ proc/13395.

[3] D. Bachiller and F. Cedó, A family of solutions of the Yang-Baxter equation, J. Algebra 412 (2014), 218-229. DOI: 10.1016/j.jalgebra.2014.05.011.

[4] D. Bachiller, F. Cedó, And E. Jespers, Solutions of the Yang-Baxter equation associated with a left brace, J. Algebra 463 (2016), 80-102. DOI: 10.1016/j. jalgebra.2016.05.024.

[5] D. Bachiller, F. Cedó, E. Jespers, And J. Okniński, A family of irretractable square-free solutions of the Yang-Baxter equation, Forum Math. 29(6) (2017), 1291-1306. DOI : 10.1515/forum-2015-0240.

[6] D. Bachiller, F. Cedó, E. Jespers, And J. Okniński, Iterated matched products of finite braces and simplicity; new solutions of the Yang-Baxter equation, Trans. Amer. Math. Soc. 370(7) (2018), 4881-4907. DOI : 10.1090/tran/7180.

[7] S. S. BEDI AND J. RAM, Jacobson radical of skew polynomial rings and skew group rings, Israel J. Math. 35(4) (1980), 327-338. DOI : 10.1007/BF02760658. 
[8] E. J. Beggs, J. D. Gould, And S. Majid, Finite group factorizations and braiding, J. Algebra 181(1) (1996), 112-151. DOI : 10.1006/jabr.1996.0112.

[9] K. I. Beidar, Y. Fong, and E. R. PuczyŁowski, Polynomial rings over nil rings cannot be homomorphically mapped onto rings with nonzero idempotents, J. Algebra 238(1) (2001), 389-399. DOI : 10.1006/jabr.2000.8632.

[10] J. P. Bell and B. Greenfeld, Free subalgebras of graded algebras, J. Algebra 483 (2017), 145-162. DOI : 10.1016/j.jalgebra.2017.04.004.

[11] J. P. Bell And B. W. Madill, Iterative algebras, Algebr. Represent. Theory 18(6) (2015), 1533-1546. DOI : 10.1007/s10468-015-9550-y.

[12] J. P. Bell, B. W. Madill, and F. Shinko, Differential polynomial rings over rings satisfying a polynomial identity, J. Algebra 423 (2015), 28-36. DOI: 10.1016/j.jalgebra.2014.09.039.

[13] J. Bergen and P. Grzeszczuk, Skew power series rings of derivation type, $J$. Algebra Appl. 10(6) (2011), 1383-1399. DOI : 10.1142/S0219498811005245.

[14] J. Bergen And P. Grzeszczuk, Skew derivations and the nil and prime radicals, Colloq. Math. 128(2) (2012), 229-236. DOI : 10.4064/cm128-2-8.

[15] J. Bergen, S. Montgomery, and D. S. Passman, Radicals of crossed products of enveloping algebras, Israel J. Math. 59(2) (1987), 167-184. DOI: 10.1007/ BF02787259.

[16] G. M. Bergman, On Jacobson radicals of graded rings, Preprint (1975). Available at http://math.berkeley.edu/ gbergman/papers/unpub/.

[17] K. A. Brown and K. R. Goodearl, "Lectures on Algebraic Quantum Groups", Advanced Courses in Mathematics, CRM Barcelona, Birkhäuser Verlag, Basel, 2002. DOI : $10.1007 / 978-3-0348-8205-7$.

[18] J. S. Carter, M. Elhamdadi, and M. Saito, Homology theory for the settheoretic Yang-Baxter equation and knot invariants from generalizations of quandles, Fund. Math. 184 (2004), 31-54. DOI : 10.4064/fm184-0-3.

[19] F. Cedó, E. Jespers, and J. Okniński, Braces and the Yang-Baxter equation, Comm. Math. Phys. 327(1) (2014), 101-116. DOI : 10.1007/s00220-014-1935-y.

[20] M. Chebotar, W.-F. Ke, P.-H. Lee, and E. R. PuczyŁowski, On polynomial rings over nil rings in several variables and the central closure of prime nil rings, Israel J. Math. 223(1) (2018), 309-322. DOI : 10.1007/s11856-017-1616-6.

[21] P. Etingof and S. Gelaki, A method of construction of finite-dimensional triangular semisimple Hopf algebras, Math. Res. Lett. 5(4) (1998), 551-561. DOI : 10.4310/MRL.1998.v5.n4.a12.

[22] P. Etingof, T. Schedler, and A. Soloviev, Set-theoretical solutions to the quantum Yang-Baxter equation, Duke Math. J. 100(2) (1999), 169-209. DOI: 10.1215/S0012-7094-99-10007-X.

[23] B. J. Gardner and R. Wiegandt, "Radical Theory of Rings", Monographs and Textbooks in Pure and Applied Mathematics 261, Marcel Dekker, Inc., New York, 2004.

[24] K. R. Goodearl, Prime ideals in skew polynomial rings and quantized Weyl algebras, J. Algebra 150(2) (1992), 324-377. DOI : 10.1016/S0021-8693(05) 80036-5.

[25] K. R. Goodearl and E. S. Letzter, Prime ideals in skew and $q$-skew polynomial rings, Mem. Amer. Math. Soc. 109(521) (1994), 106 pp. DOI: 10.1090/ memo/0521. 
[26] K. R. Goodearl and E. S. Letzter, Skew polynomial extensions of commutative Noetherian Jacobson rings, Proc. Amer. Math. Soc. 123(6) (1995), 1673-1680. DOI : $10.2307 / 2160976$.

[27] K. R. Goodearl and R. B. Warfield, JR., Primitivity in differential operator rings, Math. Z. 180(4) (1982), 503-523. DOI : 10.1007/BF01214722.

[28] B. Greenfeld, A. Leroy, A. Smoktunowicz, and M. Ziembowski, Chains of prime ideals and primitivity of $\mathbb{Z}$-graded algebras, Algebr. Represent. Theory 18(3) (2015), 777-800. DOI : 10.1007/s10468-015-9516-0.

[29] L. Guarnieri and L. Vendramin, Skew braces and the Yang-Baxter equation, Math. Comp. 86(307) (2017), 2519-2534. DOI : 10.1090/mcom/3161.

[30] C. Y. Hong, N. K. Kim, Y. Lee, And P. P. Nielsen, Amitsur's property for skew polynomials of derivation type, Rocky Mountain J. Math. 47(7) (2017), $2197-2218$.

[31] E. Jespers and E. R. PuczyŁowski, The Jacobson and Brown-McCoy radicals of rings graded by free groups, Comm. Algebra 19(2) (1991), 551-558. DOI: 10.1080/00927879108824155.

[32] P. Kanwar, A. Leroy, and J. Matczuk, Idempotents in ring extensions, $J$. Algebra 389 (2013), 128-136. DOI : 10.1016/j.jalgebra.2013.05.010.

[33] G. R. Krause and T. H. Lenagan, "Growth of Algebras and Gelfand-Kirillov Dimension", Revised edition, Graduate Studies in Mathematics 22, American Mathematical Society, Providence, RI, 2000.

[34] T. Y. Lam, "Exercises in Classical Ring Theory", Second edition, Problem Books in Mathematics, Springer-Verlag, New York, 2003. DOI : 10.1007/b97448.

[35] T. Y. Lam, A. Leroy, and J. Matczuk, Primeness, semiprimeness and prime radical of Ore extensions, Comm. Algebra 25(8) (1997), 2459-2506. DOI: 10.1080/00927879708826000.

[36] P. H. Lee and E. R. PuczyŁowski, On prime ideals and radicals of polynomial rings and graded rings, J. Pure Appl. Algebra 218(2) (2014), 323-332. DOI: $10.1016 / j \cdot j$ paa. 2013.06.004.

[37] A. Leroy and J. Matczuk, Primitivity of skew polynomial and skew Laurent polynomial rings, Comm. Algebra 24(7) (1996), 2271-2284. DOI: 10.1080/ 00927879608825699.

[38] A. Leroy and J. Matczuk, Ore extensions satisfying a polynomial identity, $J$. Algebra Appl. 5(3) (2006), 287-306. DOI : 10.1142/S0219498806001727.

[39] J.-H. LU, M. YAN, AND Y.-C. Zhu, On the set-theoretical Yang-Baxter equation, Duke Math. J. 104(1) (2000), 1-18. DOI : 10.1215/S0012-7094-00-10411-5.

[40] N. Monod, N Ozawa, And A. Thом, Is an irng singly generated as an ideal? Internat. J. Algebra Comput. 22(4) (2012), 1250036, 6 pp. DOI: 10.1142/ S0218196712500361.

[41] P. P. Nielsen And M. Ziembowski, Derivations and bounded nilpotence index, Internat. J. Algebra Comput. 25(3) (2015), 433-438. DOI: 10.1142/ S0218196715500034.

[42] E. R. PuczyŁowski And A. Smoktunowicz, On maximal ideals and the BrownMcCoy radical of polynomial rings, Comm. Algebra 26(8) (1998), 2473-2482. DOI : $10.1080 / 00927879808826292$.

[43] L. H. Rowen, "Ring Theory", Vols. I and II, Pure and Applied Mathematics 127 and 128, Academic Press, Inc., Boston, MA, 1988.

[44] W. Rump, Braces, radical rings, and the quantum Yang-Baxter equation, $J$. Algebra 307(1) (2007), 153-170. DOI : 10.1016/j.jalgebra.2006.03.040. 
[45] E. Sajsiada And P. M. Cohn, An example of a simple radical ring, J. Algebra 5(3) (1967), 373-377. DOI : 10.1016/0021-8693(67) 90048-8.

[46] A. Smoktunowicz, Polynomial rings over nil rings need not be nil, J. Algebra 233(2) (2000), 427-436. DOI : 10.1006/jabr.2000.8451.

[47] A. Smoktunowicz, On primitive ideals in graded rings, Canad. Math. Bull. 51(3) (2008), 460-466. DOI : 10.4153/CMB-2008-046-1.

[48] A. Smoktunowicz, The Jacobson radical of rings with nilpotent homogeneous elements, Bull. Lond. Math. Soc. 40(6) (2008), 917-928. DOI: 10.1112/blms/ bdn086.

[49] A. Smoktunowicz, How far can we go with Amitsur's theorem in differential polynomial rings? Israel J. Math. 219(2) (2017), 555-608. DOI : 10.1007/s11856 -017-1491-1.

[50] A. Smoktunowicz and L. Vendramin, On skew braces (with an appendix by N. Byott and L. Vendramin), J. Comb. Algebra 2(1) (2018), 47-86. DOI : 10.4171 /JCA/2-1-3.

[51] A. Smoktunowicz And M. Ziembowski, Differential polynomial rings over locally nilpotent rings need not be Jacobson radical, J. Algebra 412 (2014), 207-217. DOI : 10.1016/j.jalgebra.2014.04.022.

Be'eri Greenfeld

Department of Mathematics, Bar Ilan University, Ramat Gan 5290002, Israel

E-mail address: beeri.greenfeld@gmail.com

Agata Smoktunowicz

Maxwell Institute for Mathematical Sciences, School of Mathematics, University of Edinburgh, JCM Building, Kings Buildings, Mayfield Road Edinburgh EH9 3JZ, Scotland, UK

E-mail address: A.Smoktunowicz@ed.ac.uk

Michał Ziembowski

Faculty of Mathematics and Information Science, Warsaw University of Technology, 00-662 Warsaw, Poland

E-mail address: m.ziembowski@mini.pw.edu.pl

Primera versió rebuda el 4 de setembre de 2017, darrera versió rebuda el 13 de juliol de 2018. 